\title{
Testing ERP and MES with Digital Twins
}

\author{
Juha Hirvonen Hannu Hakalahti Mikko Ylihärsilä \\ School of Technology, Seinäjoki University of Applied Sciences, Finland, \\ \{juha.hirvonen, hannu.hakalahti, mikko.yliharsila\}@seamk.fi
}

\begin{abstract}
Enterprise resource planning (ERP) systems and manufacturing execution systems (MES) are becoming more and more important also for the small and medium-sized enterprises (SMEs). Even though the failure rates of the ERP projects seem to be exaggerated, the failures and problems in the integration cost lots of time and money. Therefore, there is clearly a need for test environments for the ERP and MES systems. This paper presents an approach to test these information systems by connecting them to the simulation models of the production and thus generating a digital twin of the production and the ERP/MES systems. As a proof-ofconcept, two different twins are constructed with two different software.
\end{abstract}

Keywords: digital twin, simulation, ERP, MES

\section{Introduction}

Enterprise resource planning (ERP) systems are integrated management systems of main business processes, such as production, input and output logistics, finance and accounting, sales and marketing, and human resources. An ERP system helps different parts of the organization to efficiently share data and information thus reducing costs and improving the management of business processes. Manufacturing execution systems (MES) are information systems for controlling, tracking, and documenting the process of manufacturing products it the factory floor. MES ensures effective production and provides information that can be utilized for improving the production output.

Traditionally, ERP and MES systems have been software for big companies. However, because of the ongoing era of digitalization and tightening competition, also small and medium-sized enterprises (SME) are more commonly investing in these systems (Supramaniam et al, 2014).

ERP projects are notorious for having low success rates and swollen costs, and there are numerous web pages by different consultant firms repeating the doomsday figures that assure that the frightening reputation is true. However, most of them do not have any reference for their figures and the ones that have seem to be cross-referencing each other or not-existing reports. Nevertheless, there are some actual publications by the consultant companies as well. According to a report by McKinsey, roughly $75 \%$ of ERP projects fail to stay either on schedule or within budget, and around $67 \%$ have a negative return on investment (Casanova et $a l, 2019)$. However, the report does not mention the exact source of these figures. Another report by Panorama Consulting Group (2020) states that $38 \%$ of the ERP projects exceed the budget (average $66 \%$ over) and $47 \%$ do not stay in the schedule (average $33 \%$ overtime). The report is based on a survey with 181 respondents, out of which $34 \%$ are manufacturers (Panorama Consulting Group, 2020). Yet another report made by Mint Jutras (2019) states that $46 \%$ of the ERP projects exceed the budget and $38 \%$ do not stay in the schedule. It also states that $67 \%$ rate their implementation as successful. The report is based on a survey of 315 respondents. These figures are much more positive than the ones reported by e.g. McKinsey and fairly in line with the ones presented by Panorama Consulting Group. However, the authors also suspect that based on the actual benefits realized, many of the respondents might be overrating their success (Mint Jutras, 2019).

Some recent academic research has been conducted on the success of the ERP projects as well. Johansson and Sudzina (2019) studied the ERP implementations in the European SMEs and they found that only $26 \%$ exceeded the budget, of which around one third exceeded the budget with $50 \%$ or more. The survey had 121 respondents from Denmark, Slovakia and Slovenia (Johansson and Sudzina, 2019). As stated by Haddara and Zach, (2012), the ERP implementation projects are typically easier and shorter in SMEs since the size of the organization is smaller and the business complexity lower. However, the staff must often play multiple roles in small companies, and thus allocating enough resources to the ERP project might be problematic (Mint Jutra, 2019). Also, for the SME, the overruns in the budget and time can be even more disastrous than for a big company.

Based on the surveys presented, the failure rates of the ERP projects seem to be overrated. Nevertheless, the budget and schedule overruns are quite common. Also, the unchallenged fact is that some extremely expensive ERP implementation catastrophes have occurred (e.g. listed in Ram et al, 2013), and many times the employees have found the fresh ERP system to be too 
stiff and to lead to some inefficient practices such as process duplication (Kerr and Houghton, 2014). To support the implementation process and to estimate the possible bottlenecks, different tools and analyses such as critical success factors (Ram et al, 2013), critical success strategies (Yeh and $\mathrm{Xu}, 2013$ ), critical failure factors (Amid et al, 2012), project risk modeling (Aloini et al, 2012), and maturity models (Kerr and Houghton, 2014) have been developed. However, these approaches may be too theoretical and too complex especially for the needs of the SMEs. More concrete testing procedures would be beneficial.

The actual testing of the ERP and MES systems in the production environment before the investment decision is extremely difficult. Often, the only option is to trust in the supplier's promises. In this paper, a testing approach based on digital twins is suggested.

Barricelli et al (2019) define digital twins as computer-based models that are simulating, emulating, or mirroring a physical entity or a process. They emphasize that instead of "just" a simulation, it is the virtual counterpart of the entity or the process, and the data flow in and out of the twin is essential. Manufacturing is one of the main application domains of the digital twins, and the application cases include factory design, manufacturing optimization, consistent process monitoring, and predictive maintenance (Barricelli et al, 2019). Thus, it seems a promising technology also for testing the IT systems for the manufacturing companies.

In this paper, a proof-of-concept of using digital twins to test ERP and MES systems is presented. The main target group of the approach is SMEs. The digital twins are constructed by generating the simulation models of two different manufacturing systems and connecting them to the custom MES systems and an ERP system with an online shop interface. This way, handling the customer order, starting the production, monitoring the production process, and receiving data to the IT systems from the production can be experimented.

The rest of the paper is organized as follows: Section 2 discusses the pilot production environments used in this study, Section 3 presents the digital twins produced, and Section 4 draws the conclusion.

\section{Pilot Production Environments}

This section presents the two manufacturing systems that were used as pilots in this study. They were the educational cyber-physical factory and a flexible manufacturing system (FMS) cell. The systems are presented in their sub-sections.

\subsection{Educational Cyber-Physical Factory}

The cyber-physical factory is a modular production line for education purposes made by Festo Didactic. The factory assembles simple cell-phone-like products consisting of a back cover, a printed circuit board (PCB) and fuses. The PCBs are made of plastic and they do not have conductive tracks, pads or connectors - only the holders for the fuses. The purpose of the cyber-physical Factory is to demonstrate different assembly processes and hardware as well as offer insight into the modular design, networked logistics, and MES integration. Altogether 192 different product variants can be assembled from the components of different colors.

The cyber-physical factory consists of a high-bay storage for the back covers and the assembled products, a drilling station, a robot assembly cell, in which the robot mounts the PCBs to the back covers and the fuses to the PCBs, and a machine-vision-based inspection station for assuring the correct composition of the assembled product. The factory is controlled by Festo's own MES4 software. A custom MES software made by the students is in use as well. Figure 1 presents the cyber-physical factory, and Figure 2 shows an example of a product that can be assembled with the factory.

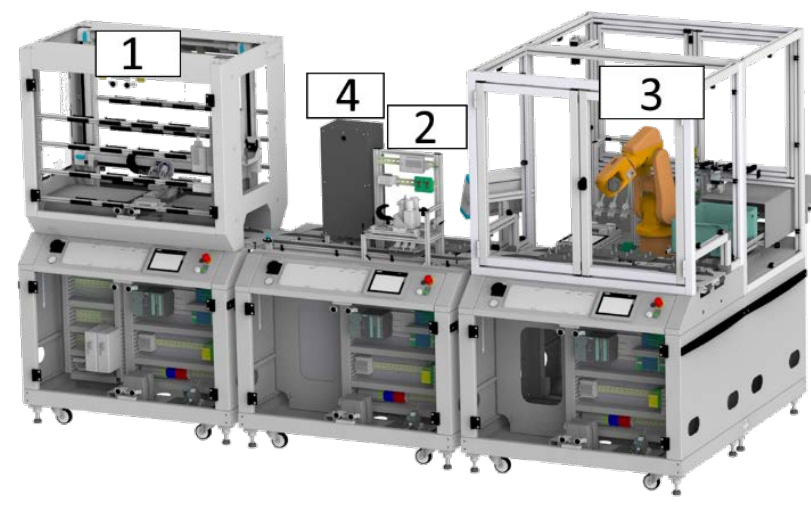

Figure 1. The educational cyber-physical factory. The high-bay rack (1), the drilling station (2), the robot assembly cell (3), and the machine vision station (4). The modules are numbered in the order of the process flow.

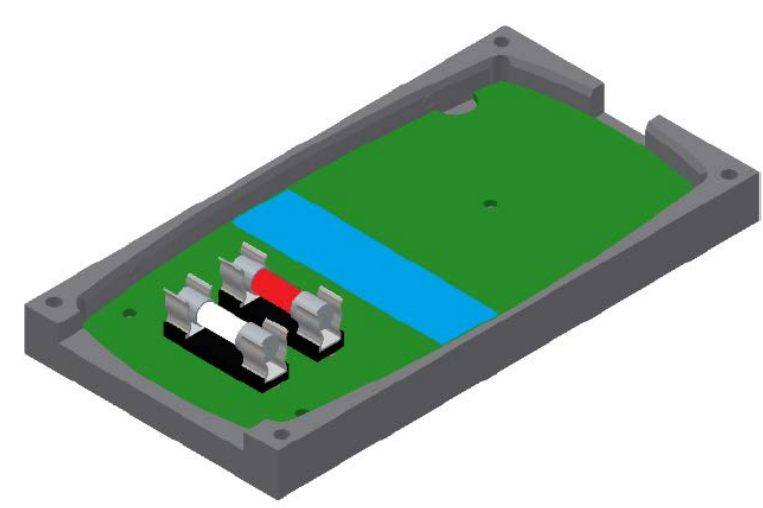

Figure 2. One product variant of the cyber-physical factory consisting of a gray back cover, a blue PCB and transparent and red fuses. 


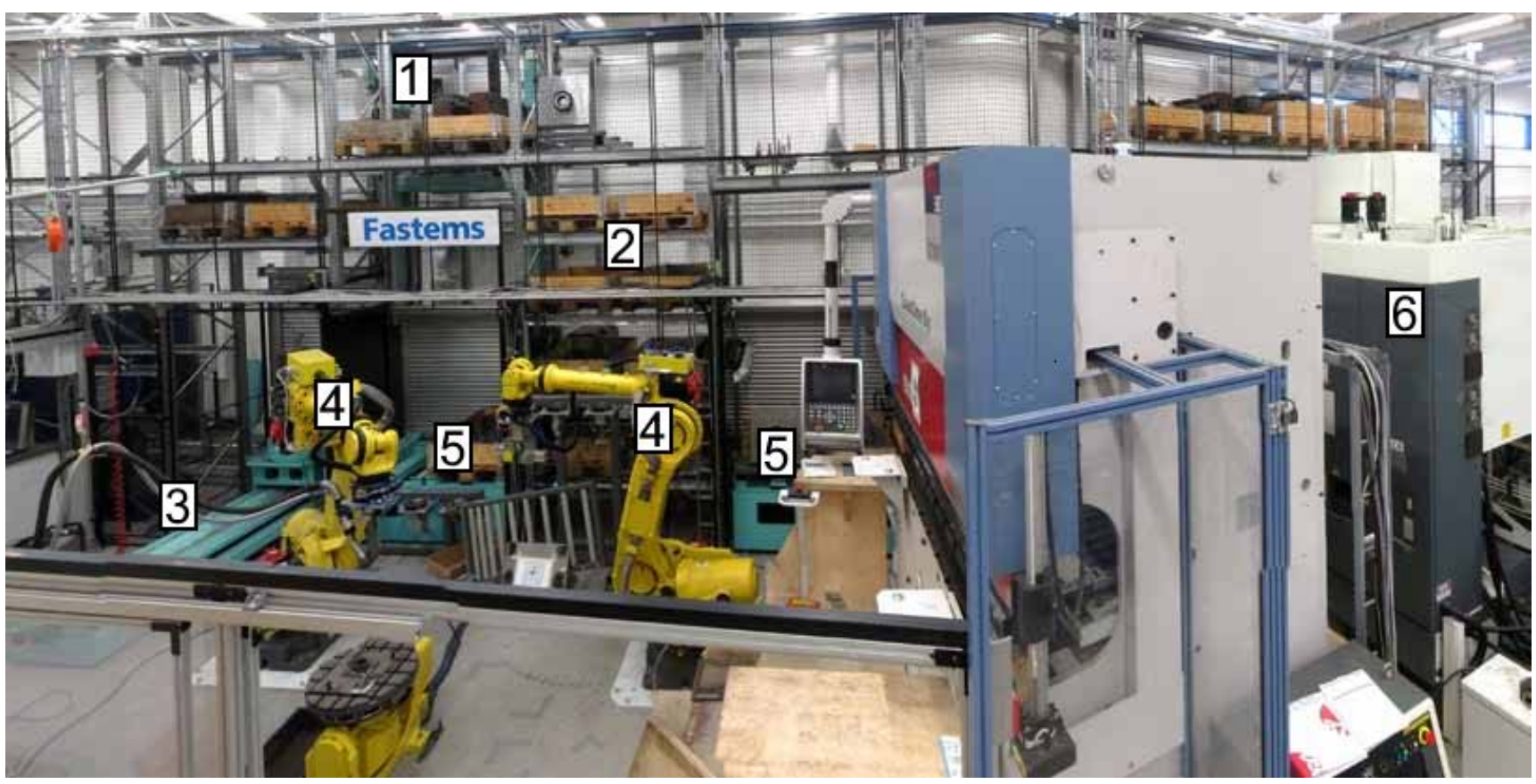

Figure 3. The FMS cell. The stacker crane (1), the pallet storage (2), the loading station (3), the industrial robot (4), the pallet stations (5), and the machining center (6).

\subsection{FMS Cell}

The flexible manufacturing system (FMS) cell by Fastems consists of a stacker crane, a pallet storage for wooden Euro-pallets and machining pallets, a loading station for the machining pallet, an industrial robot, pallet stations inside the robot cell, and a machining center. The MES system used to control the production of the cell is Fastems MMS. The FMS cell can be configured to produce a high variety of products, and the cell is manufacturing support parts in the example application of this study. The production cycle has six phases, and it goes as follows: (1) The stacker crane picks a pallet containing the blank and an empty pallet from the pallet storage and places them into the pallet stations of the robot. (2) The robot picks the blank from the pallet in the pallet station and places it to the machining pallet in the loading station. (3) The stacker crane takes the machining pallet and the blank to the machining center, where the support part is machined from the blank. (4) The stacker crane returns the machining pallet and the machined support part to the loading station after machining. (5) The robot picks the support part from the machining pallet and places it to the machine vision inspection. (6) The robot places the support part to the pallet for the machined parts after the inspection. Figure 3 shows the FMS cell.

\section{Digital Twins}

This section presents the digital twins of the production environments discussed in the previous section. The ERP system used with both the digital twins was Odoo. Odoo is an open-source ERP system and it was chosen because it is attractive for SMEs due to its affordable price (community version: free) and the high availability of different modules and add-ons that make the software scalable. The users can also create their own Odoo modules.

In order to send the manufacturing orders from Odoo to the simulation models, it was necessary to develop a small Odoo application for this purpose. In practice, the Odoo application reads the sale order data, creates a manufacturing order, and sends it to an external Python application.

\subsection{Python web application}

The Python web application acts as an intermediary between Odoo and the simulation models. It receives the manufacturing order from the Odoo, validates the order data, creates the steps of the work rotation, and sends commands to the simulation models. It also receives data from the simulation models. The application communicates with the simulation models via TCP/IP sockets and the data is sent and received in JSON format.

The application has a user interface in the form of a web page, where the user can inspect and get information from the states of the devices in the models, monitor the storage inventories, run the individual subprogram of the devices, create new manufacturing orders, and control (start/delete) pending manufacturing orders. Thus, the application can be considered as an MES tailored for the simulation models.

The application is implemented with Flask micro web framework and the web page uses Bootstrap 4 CSS framework for the visual appearance. 


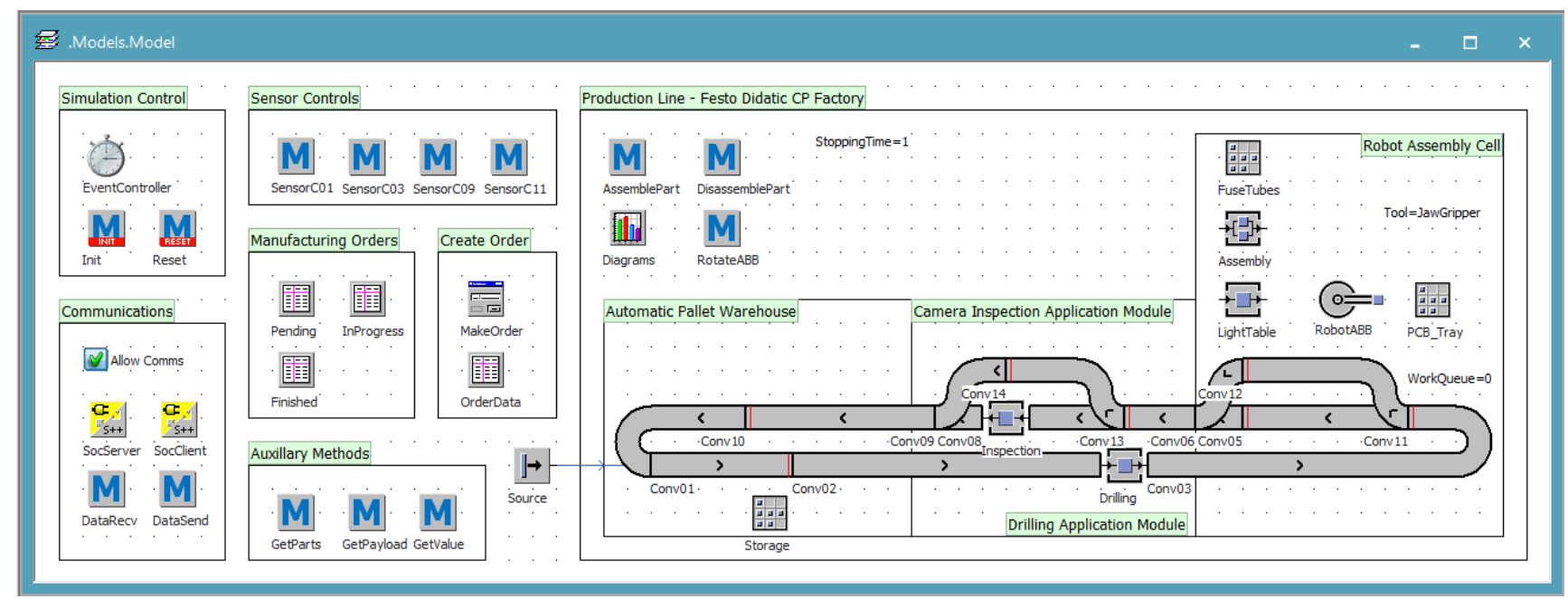

Figure 4. The digital twin of the production process of the cyber-physical factory.

\subsection{Educational Cyber-Physical Factory}

The simulation model of the Cyber-Physical Factory was made with Tecnomatix Plant Simulation, and the manufacturing orders are created with the Odoo ERP system. The product variants are generated in the Siemens Teamcenter PDM system from where a Python accessory inserts them into the Odoo database. In the database, a bill of materials (BOM) is created for the product and the product is published in the Odoo online shop. In the online shop, the customer can choose the colors (and in the case of the fuses, the number) of the components and make the order.

The Plant Simulation model assembles the product as well as sends information about the state of the assembly process and the current storage inventories back to the web application. Figure 4 presents the digital twin of the production process of the cyber-physical factory, and Figure 5 shows the view from the Odoo online shop.

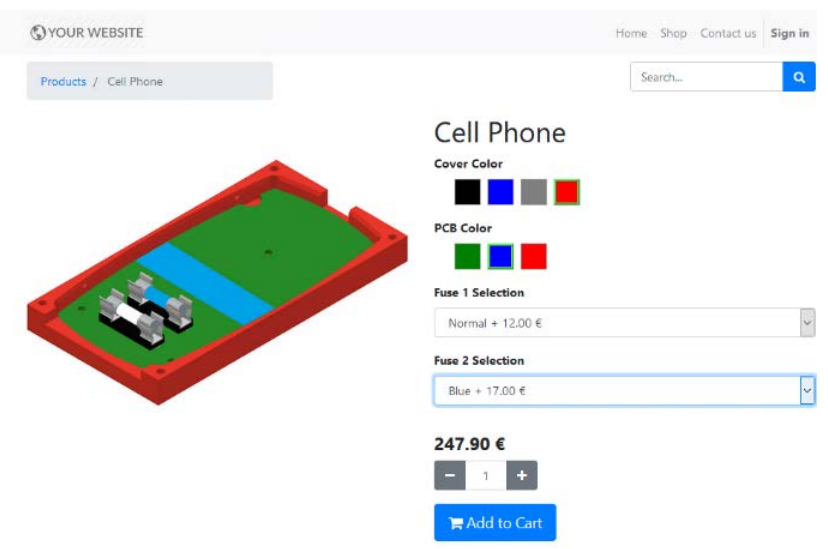

Figure 5. View from the Odoo online shop for ordering the "cell phones".

\subsection{FMS Cell}

The simulation model of the FMS cell was made with Visual Components. The industrial robots, workpiece positioner, Euro-pallets and shelves were added from the model library of the Visual Components while the rest of the devices and accessories were designed in Siemens NX. The CAD files were then imported into the Visual Components where the properties of the devices were defined.

In Visual Components, the functionality of the devices can be extended with scripts that are written in the Python 2.7 programming language. In the simulation model of the FMS cell, a Python script enabling a TCP/IP connection to an external Python web application was added to the stacker crane, the loading station, the robot and the machining center. Thus, these devices can send and receive data.

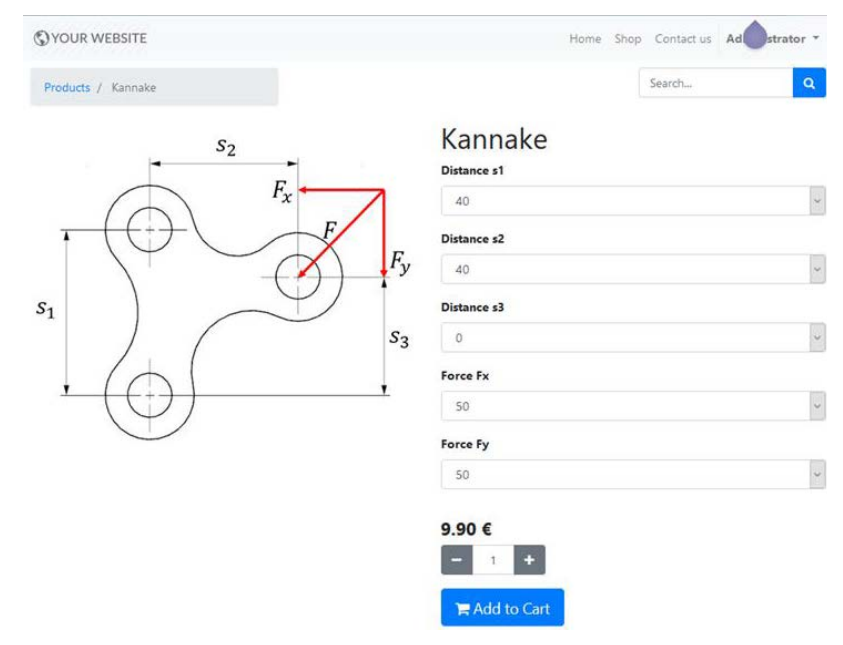

Figure 6. View from the Odoo online shop for ordering the support parts. 


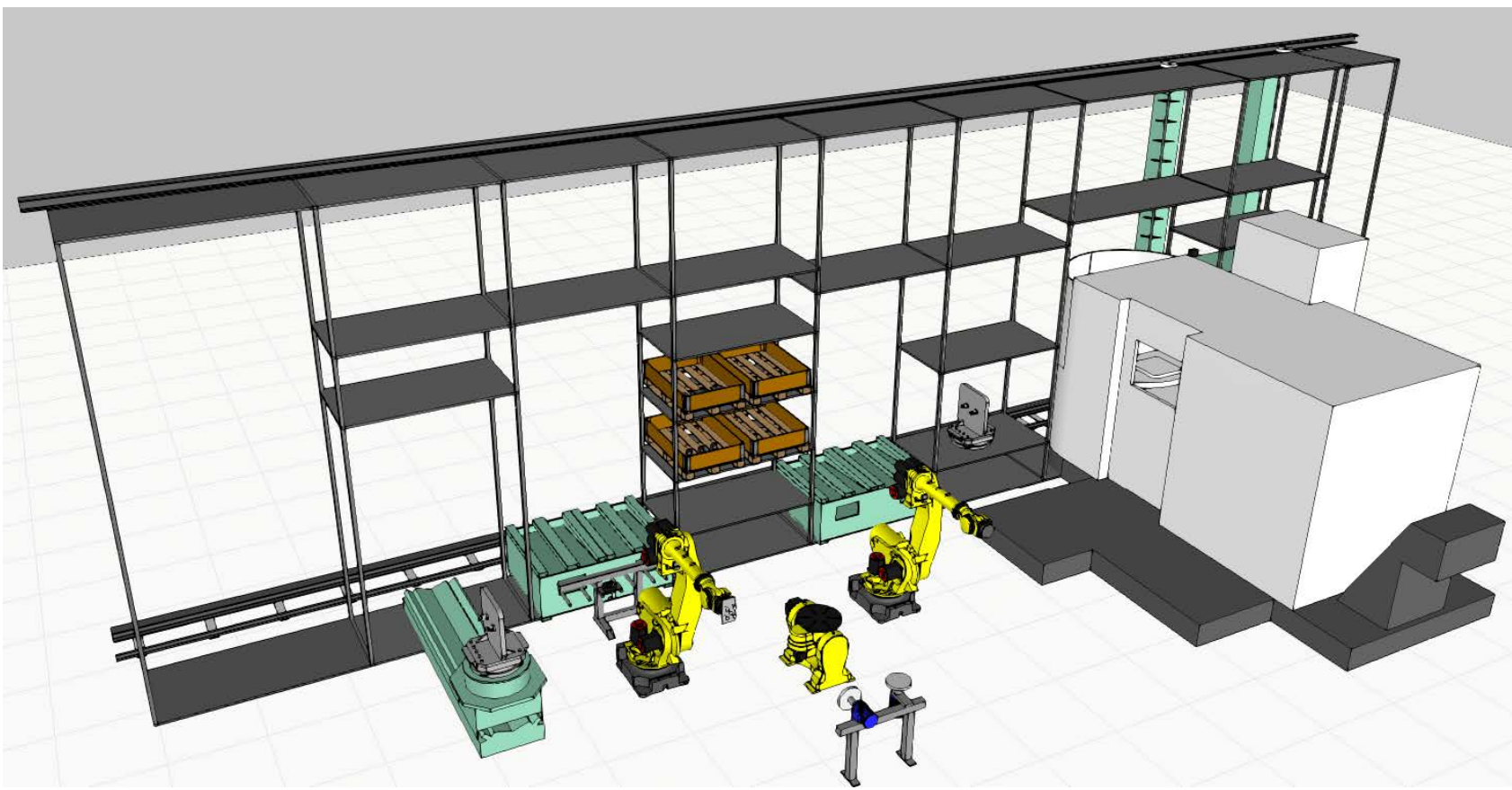

Figure 4. The digital twin of the FMS cell.

The simulation model of the FMS cell can run two work rotations: machining of a support part and polishing a cube. In the Odoo online shop, the customer can set the distances of the holes of the support, and the $\mathrm{X}$ and $\mathrm{Y}$ components of the force acting to the support. The machining time of the support is calculated based on these parameters. During the machining work rotation, the machining time is sent to the machining center.

Figure 6 shows the view from the online shop, and the digital twin of the FMS cell is seen in Figure 7.

\section{Results and Discussion}

The digital twins of the educational cyber-physical factory and the FMS cell were built and connected with the Odoo ERP system as discussed in Chapter 3. Also, the physical cyber-physical lab was connected with the Odoo ERP. Placing customer orders in the online shop as well as accepting the orders and starting the production in the admin side are functioning similarly with the physical system and the digital twin. The FMS cell will also be connected with Odoo in the near future. So far, the tests have been limited to creating individual device interfaces to the industrial robot and the machining center. The simulation models mimic the work cycles of their physical counterparts, but all the details were not modeled - for example, the shape of the ready support part in the digital twin of the FMS cell is always similar and do not imitate the ordered part. However, all the tests described here offer a proof-ofconcept of the approach.

Apart from testing the ERP-MES integration, this approach could be utilized to test the capacity to produce different kinds of big orders and estimate the delivery time. Also, these kinds of digital twins can be used widely for educational purposes. This is resource efficient as a big group of students can work simultaneously with the models instead of working in shifts in one laboratory. The model of the FMS cell presented in this paper has already been utilized in teaching e.g. socket programming. Currently, the simulation models lack the functionality to inform the MES or ERP of an error that occurs in the production, but this kind of functionality can be added. This addition would give also valuable information about the data transfer from the error situations of the production to the information systems.

One important aspect is the modularity of the presented approach. This is estimated below separately for both twins.

The digital twin of the educational cyber-physical factory consisted of the Odoo online shop, the PDMOdoo interface, the Odoo-Plant Simulation interface and the Plant Simulation model. If some company would like to test the suitability of Odoo to their production, the Plant Simulation model and the PDM-Odoo interface should be fully reconstructed. However, the PDM-Odoo interface is not compulsory as the product variants can be created in Odoo itself in the new versions (Odoo 13 or above). The Odoo-Plant Simulation interface would need to be updated but much of it could be reused. On the other hand, if a different ERP system should be tested with the re-existing model, the ERPPlant Simulation interface should be reconstructed.

The digital twin of the FMS cell consisted of Odoo online shop, Odoo-Visual Components interface (the 
REST interface), Visual Components model and the TCP/IP socket communication blocks inside the model. Again, if the production scheme changes, the Visual Components model needs to be reconstructed. The Odoo-Visual Components interface would need an update but many of its parts would be still usable. The socket communication blocks would need no more than a minor update. Changing the ERP system would need reconstructing the ERP-Visual Components interface. It is also worth noting, that the Odoo-Plant Simulation interface and the Odoo-Visual Components interface are mostly similar instead of few conditional statements. The major reason for the differences in the interfaces is the difference in functioning of the physical devices: the modules of the educational cyber-physical factory poll actively the MES whereas the devices of the FMS cell just wait for the orders from the MES.

\section{Conclusion}

This paper presented the concept of testing ERP and MES integration with a digital twin approach. The benefit of the approach is that it enables testing the ERPMES integration beforehand without the need to interrupt the actual production. The approach gives a concrete demonstration of the functioning of the ERP system. Therefore, making the order in a online shop, handling orders, and the exchange of the data between the systems can be tested with it. Two different simulation models were created with two different modeling software to show the flexibility of the method.

The main downside of the approach is that generating a digital twin takes time. If the production system of the company is very complex, then a highly complex model must be constructed as well. However, as the target group of the method is SMEs, the production is not expected to be extremely complicated. Another disadvantage is that the interfaces between the model and the ERP/MES systems should be tailored for each ERP system. Yet, the selection of the ERP systems suitable for SMEs is not that large and thus this problem is not that crucial. Lastly, the constant question with the models is how well they correspond to the actual system. Precision is needed in generating the digital twins.

Although there are some open questions, the demonstrations of the digital twin approach to test ERP and MES are really promising. Another ERP system and some commercial MES systems will be tested in the near future. Future work includes also adding more intelligence to ordering and manufacturing. Firstly, the support part orders made for the FMS system will be passed to a CAD program and further to FEM calculation to prevent the user from ordering a physically inconsistent part. Furthermore, the geometry of the support part will be fed into CAM software for automatic CNC cutting program generation. The generated CNC program will be simulated to validate for collision-free path and calculate the part's machining time. Calculated machining time will be further used to fine-tune the full factory simulation times, calculating more precise cost estimations and price quotations.

\section{Acknowledgements}

This paper has been written as a part of the project Enterprise Digital Twin Platform, and the funding from the Regional Council of South Ostrobothnia is greatly appreciated.

\section{References}

Davide Aloini, Riccardo Dulmin, and Valeria Mininno. Modelling and Assessing ERP Project Risks: A Petri Net Approach. European Journal of Operational Research, 220(2):484-495, 2012. doi: 10.1016/j.ejor.2012.01.062.

Amin Amid, Morteza Moalagh, and Ahad Zare Ravasan. Identification and Classification of ERP Critical Failure Factors in Iranian Industries. Information Systems, 37(3):227-237, 2012. doi: 10.1016/j.is.2011.10.010.

Barbara Rita Barricelli, Elena Casiraghi, and Daniela Fogli. A Survey on Digital Twin: Definitions, Characteristics, Applications, and Design Implications. IEEE Access, Vol 7, pp. 167653-167671, 2019. doi: 10.1109/ACCESS.2019.2953499.

Didier Casanova, Swati Lohiya, Jerome Loufrani, Matteo Pacca, and Peter Peters. Agile in Enterprise Resource Planning: A Myth No More. McKinsey Digital, 2019.

Moutaz Haddara, and Ondrej Zach. ERP Systems in SMEs: an Extended Literature Review. International Journal of Information Science, 2(6):106-116, 2012. doi: 10.5923/j.ijis.20120206.06.

Björn Johansson, and Frantisek Sudzina. Actual vs. Planned ERP Systems Implementation Costs in European SMEs. Proceedings of the European Conference on Information Management and Evaluation, pp. 232-238, 2019.

Don Kerr, and Luke Houghton. The Dark Side of ERP Implementations: Narratives of Domination, Confusion and Disruptive Ambiguity. Prometheus, 32(3):281-295, 2014. doi: 10.1080/08109028.2015.1017247.

Mint Jutras. The Real Facts about ERP Implementation: Busting the Myth of Failure, But Are You Overrating Your Success? Ultra Consultants, 2019.

Panorama Consulting Group. The 2020 ERP report, 2020.

Jiwat Ram, David Corkindale, and Ming-Lu Wu. Implementation Critical Success Factors (CSFs) for ERP: Do They Contribute to Implementation Success and Postimplementation Performance? International Journal of Production Economics, 144(1):157-74, 2013. doi: 10.1016/j.ijpe.2013.01.032.

Mahadevan Supramaniam, Azween Abdullah, and Ramachandran Ponnan. Cost Analysis on ERP System Implementation amongst Malaysian SMEs. International Journal of Trade, Economics and Finance, 5(1):72-76, 2014. doi: 10.7763/ijtef.2014.v5.343.

Chung-Hsing Yeh, and Yan Xu. Managing Critical Success Strategies for an Enterprise Resource Planning Project. European Journal of Operational Research, 230(3):604614, 2013. doi: 10.1016/j.ejor.2013.04.032. 